\title{
Las conversaciones en las organizaciones: reto investigativo ante la evidencia de los paradigmas presentes
}

\author{
(ล) \\ Luz Marina Vélez Jiménez** \\ Carlos Iván Ruiz Roldán \\ Claudia Mónica Zuleta Ruiz
}

Recibido: 20 de febrero de 2012 Aprobado: 27 de marzo de 2012

\begin{abstract}
Resumen
El lenguaje es invención y constitución de realidad; las organizaciones, como cualquier otro fenómeno social solo existen porque ocupan un lugar en el lenguaje. Las organizaciones están conformadas por personas, van más allá de ser un conjunto de roles institucionalizados; se constituyen en espacios simbólicos comunes, creando nuevos significados. Conversar implica un ejercicio de autonomía, autocontrol, autoridad, autoestima y autodisciplina; es decir, un ejercicio autodidacto. En ese sentido, las organizaciones pueden definirse como redes de conversaciones que se auto-constituyen.
\end{abstract}

En la primera fase del proyecto (ver nota al pie), se rastrearon diversas fuentes de información, entre ellas las de generalización y las históricas. Como hallazgos, se evidencian los estudios de Pablo Antonio Múnera y Uriel Sánchez Zuluaga, Martha Lucía Gómez, Ana Isabel Martínez, Juliana Vélez y Alejandra Rúa, entre otros.

Dado el incipiente alcance de los resultados de dichos estudios, se evidencia la necesidad de realizar nuevas investigaciones en las que el objeto específico sea la conversación organizacional, puesto que constituye, para la Comunicación como disciplina académica, un campo nuevo de indagación en el ámbito nacional e internacional.

Palabras clave: organización, comunicación, conversación, relación, transformación, fenomenología.

\footnotetext{
Este artículo tiene origen en la primera fase del proyecto "Las conversaciones organizacionales al interior de las empresas en la ciudad de Medellín", desarrollado en la línea de investigación "ORGANIZACIONES, RELACIONES y COMUNICACIONES", por el grupo de investigación OTRO SENTIDO, del programa de Comunicación Organizacional de la Colegiatura Colombiana, que corresponde al estado del arte de las investigaciones acerca del concepto de "conversación" como objeto. El proyecto está financiado con recursos institucionales.

* Magíster en Filosofía de la Universidad Pontificia Bolivariana, Medellín-Colombia. Antropóloga de la Universidad de Antioquia, MedellínColombia. Actualmente se desempeña como docente investigadora de temas socio-culturales en la Colegiatura Colombiana, MedellínColombia.Correo electrónico: luzmarina.velez@colegiatura.edu.co

*** Magíster en Cultura de los Medios de Comunicación, University of Strathclyde, Escocia. Psicólogo de la Universidad de San Buenaventura, Medellín-Colombia. Docente Investigador en la Colegiatura Colombiana, Medellín-Colombia.

... Candidata a magíster en Comunicaciones de la Universidad de Antioquia, Medellín-Colombia. Historiadora de la Universidad Nacional, Medellín-Colombia. Especialista en Gestión y Promoción Cultural de la Universidad de Antioquia, Medellín-Colombia. Actualmente se desempeña como docente en la Colegiatura Colombiana.
} 


\title{
Conversations within Organizations: A Research Challenge before the Evidence of Current Paradigms
}

\begin{abstract}
Language is invention and constitution of reality; organizations, as any other social phenomenon, do not only exist because they occupy a place in language. Organizations are formed by people and are more than a number of institutionalized roles; organizations become common symbolic spaces by creating new meanings. Conversations imply the exercise of autonomy, self-control, authority, self-esteem, and self-discipline; that is, a self-taught exercise. Organizations can then be defined as a network of self-constitution conversations.

Several information sources, such as generalization and historical sources, were traced during the first stage of the project (see footnote). Findings include studies conducted by Pablo Antonio Múnera and Uriel Sánchez Zuluaga; Martha Lucía Gómez; Ana Isabel Martínez; Juliana Vélez and Alejandra Rúa; among others.

Given the incipient scope of results found in such studies, a clear evidence of a need for conducting new researches where the specific objective is "organizational discussions" is observed, since it becomes a new field of national and international research for Communication as an academic discipline.
\end{abstract}

Key words: Organization; communication; conversation; relationship; transformation; phenomenology. 


\section{Consideraciones iniciales}

"La conversación es la estética de la comunicación".

Humberto Maturana

La conversación es una circunstancia de múltiples sentidos, que convierte en objetos de estudio a la sensación, la expresión, la figuración, la abstracción y la temporalidad humanas. Es una herramienta, representación en movimiento; es el crisol de la necesidad y de la memoria humana. Es la confirmación de que el hombre es en tanto está en el lenguaje. En términos espirituales, la conversación es una cuestión de "cada cual consigo mismo"; en términos organizacionales, de "nosotros" con "nosotros" y, en términos sociales, de "nosotros" con los "otros".

Independientemente de que las conversaciones sean orales o escritas, estas "visibilizan", ocultan o traslapan sujetos en relación, que de alguna manera evidencian la esencia de lo oral ${ }^{1}$; en este sentido, aparecen el mirar, el escuchar, el sentir y el comprender al "otro" con el que se conversa, como partes de la moralidad de una conversación. Conversar implica un ejercicio de autonomía, autocontrol, autoridad, autoestima, autodisciplina; es decir, un ejercicio autodidacto -en términos de instrucción y construcción del sí mismo-; y también entraña el riesgo de convertirse en una práctica o conducta autoflagelante, autócrata, autómata y automática. Desde la cultura, cultivare ("hacer crecer") y desde la creatividad, creare ("engendrar", "transformar"), una conversación puede ser un escenario, un objeto y una acción (reales y simbólicos) para cuidar de sí, de los otros, de las cosas, e incluso, de lo sagrado -en tanto búsqueda de trascendencia-. La conversación como posibilidad expansiva, crea-

1 El vocablo "oral" viene de "orar", y este del latín orare: "hablar públicamente en vos alta", "expresarse"; también "rogar", "solicitar", "dar un discurso", "rezar". De este verbo nos llega "oración", "orador", "oráculo"; "adorar", "adorable", "adorador", "adoración" (reconocer al otro). Desde la palabra oralis (derivado, a su vez, del indoeuropeo os "boca" y el sufijo español al "de", "relativo a") podemos asociar la idea de transmitir o entregar mediante las facultades propias o pertenecientes a la boca; es decir, la "comunicación por la palabra" (N de los A). tiva y cultural del ser humano es una forma de "autoadherirse al mundo", a su lógica, a su praxis; porque en la pregunta "¿cómo conversamos?" se deja ver nuestra humanidad virtuosa y viciosa; y en la pregunta "¿cómo conversar mejor?" aparece como el "sentido de los sentidos" el convivir práctico, responsable y amoroso (en términos de "política universal"). De esta manera, la conversación es una zona de contacto que da cuenta de nuestra identidad hecha palabra y silencio; es una práctica interpretativa; un espacio de copresencia; un discurso que marca los modos de ser, hacer y saber; una estrategia organizativa, organizacional y organizada, donde como práctica ética y estética, el hombre instaura discursos de las historias mínimas y máximas del relacionarse con lo "otro" contemporáneo.

Las conversaciones viven en el lenguaje, y en el lenguaje los hombres, transformando su realidad histórica cultural.

\section{Reseña evolutiva de las conversaciones}

"Las conversaciones inteligentes requieren individuos capaces de reflexionar sobre sus propios pensamientos".

Peter Senge

La comunicación a través del lenguaje verbal es una construcción cultural de los seres humanos; una modalidad de uso de ese lenguaje es la conversación, que consiste en una interacción oral entre dos o más personas e incluye, además de la palabra hablada, los gestos, las miradas y los silencios: "(...) La capacidad de articular los sonidos, sin dificultades anatómicas o fisiológicas, comenzó hace solamente 120.000 años (...) Se considera que los lenguajes hablados y la lengua que recurre a la doble articulación aparecen al mismo tiempo" (Serrano, 2007: 173).

Las conversaciones suceden en todos los espacios sociales (la familia, la escuela, las empresas, la calle; en cualquier lugar donde interactúen los seres humanos): "(...) La conversación 
es, por así decirlo, la 'forma fundamental' de la interacción oral y a la vez un componente esencial del trato cotidiano -es decir, no específico ni especializado- de las personas en situaciones sociales" (Van Dijk, s.f).

A través de la historia de la humanidad, la conversación ha tenido diversos significados y representa los sistemas culturales de una sociedad, como los códigos, los signos y los símbolos.

A. Briz (citado por Alonso-Cortés Fradejas, 1998), por su parte, afirma que, frente a otro tipo de discursos hablados, la conversación se caracteriza por tratarse de una interlocución en presencia (cara a cara), inmediata y actual, en la que la toma de turno no está predeterminada y se produce de una forma dinámica, y que es cooperativa en cuanto al tema de conversación y la intervención del otro. Estos son para él no solo rasgos que permiten reconocer el discurso conversacional, sino condiciones necesarias para hablar de conversación y además 'un principio de tipología discursiva'.

La conversación es la forma más cotidiana de comunicación humana, solo se requieren dos personas y la disponibilidad para hacerlo; se puede realizar de la manera más simple o más compleja. Desde la convivencia familiar hasta las formas de operación política, social y cultural, la conversación, la mayoría de las veces, se hace para llegar a acuerdos. Según Maturana:

La historia evolutiva de nuestro linaje, como una historia de la conversación de una tendencia neoténica en la biología del amor, es una historia de vida social también centrada en la consensualidad y la cooperación, no en la competitividad o la lucha agresiva. Como tal, nuestra historia evolutiva es una historia de expansión de las capacidades para lograr consensualidad, y de ahí la expansión de la inteligencia (...) Las culturas son redes de conversaciones (Maturana Romesín, 2002: 224).

En el texto La República, Platón dice que la dialéctica "de por sí no significa más que 'arte de la conversación, del diálogo o de la discusión' y como tal designa aquella destreza o habilidad que los jóvenes ponen gran empeño en adquirir para medrar en la vida pública o lucirse en la privada" (Platón, 2003: 18). Y agrega: "(...) El arte del diálogo y de la discusión no son otra cosa que el ejercicio adecuado de la razón para el descubrimiento de la verdad, es decir, del mundo de las ideas y, en último término, de la idea suprema del bien" (Platón, 2003: 98).

Después de la aparición de la imprenta en Occidente (1450) se incrementaron los espacios públicos de conversación -las tabernas, los baños y los cafés-. En el siglo XVII había 600 cafés en Estambul y en Londres 500; en estos espacios se intercambiaban los últimos acontecimientos sobre comercio, temas científicos y artísticos: "Clubes y cafés inspiraron la creación de comunidades imaginarias de comunicación oral" (Burke, 2002: 43). La proliferación de libros contribuyó así al incremento de las conversaciones.

Las formas de conversación han cambiado desde la invención de los primeros instrumentos tecnológicos para comunicarse (el teléfono, por ejemplo, posibilitó la ilusión de cercanía en la conversación con el "otro" como interlocutor). En la actualidad, las llamadas TIC (Tecnologías de la Información y la Comunicación) han transformado las conversaciones (como lo evidencia el uso de los celulares, los blackberries, y el chat, por ejemplo), pues la presencia de este tipo de adminículos ha afectado de tal manera las interacciones y las relaciones entre personas y los grupos a los cuales estas pertenecen, que han llegado a reemplazar, incluso, el primigenio contacto "cara a cara".

La conversación convencional es la forma más elemental de la conservación y del control de las estructuras sociales en las interacciones "de micro-nivel", es decir, en el nivel de la relación directa entre participantes (Van Dijk, s. f.). Hay una urgencia actual de abordar las conversaciones como objetos de estudio puntuales, sobre todo en el ámbito de las organizaciones 
humanas, las cuales son también ahora, a su vez, objeto de estudio interdisciplinario.

\section{Las organizaciones y las conversaciones}

El mundo de hoy es un mundo de organizaciones. Como dice Rubén Dittus (s. f.), no se puede entender el valor de lo humano sin las organizaciones. La definición clásica de organización está en camino de ser superada ("un conjunto de individuos con metas comunes y con normas y roles institucionalizados"; es decir, personas que cooperan para satisfacer deseos individuales y colectivos). De acuerdo con Dittus, (s. f.) "la vida organizacional es una vida de cooperación, y por ende, de comunicación. No son separables ambos fenómenos: la organización no se entiende sin la comunicación, y viceversa. (...) La organización permite o no la comunicación".

Según la idea anterior, la realidad de las organizaciones es una suma de espacios simbólicos comunes al entorno psíquico, donde se crean nuevos significados entre y para los miembros de dicho entorno, y no un conjunto de "artefactos" o de roles institucionalizados. La organización es, entonces, una red de conversaciones (Flores (s. f. citado en Dittus, s. f.), una red de comunicación (o de códigos comunicables) que se autoconstituye, no como un conjunto de seres humanos, sino de compromisos lingüísticos; una red de actos del habla, que ubica a las personas como parte de su entorno. De ese modo, la organización tiene en cada una de ellas una presencia psíquica. Esta postura se sustenta concibiendo la conversación como "la unidad mínima de interacción social, orientada hacia la ejecución con éxito de acciones" (Flores (s. f. citado en Dittus, s. f.). De este modo, la conversación se convierte en un fenómeno clave en las organizaciones, partiendo de la base de que el lenguaje es invención y constitución de realidad: las organizaciones, como cualquier otro fenómeno social, solo existen porque ocupan un lugar en el lenguaje.

El énfasis del concepto está centrado en la "comunicación para la acción"; es decir, lo que crea una organización - una red de conversaciones- son actos comunicativos con el fin de ver resultados concretos en el ámbito de los negocios, de la educación, del tiempo libre, de las finanzas o de la política. (Dittus, s.f.). Para este caso, las empresas, como una forma de organización, constituyen un claro ejemplo del poder del lenguaje, del poder de las conversaciones.

En el ámbito sociocultural existen tensiones que lesionan y desvirtúan el "estar juntos", imperativo al que se ven abocados los individuos al pertenecer a alguna organización de cualquier índole. Esto implica una nueva postura ética en la que las organizaciones deben promover relaciones en las que el poder sea dinamizado de una manera sinérgica; es decir, que al tiempo que consigan sus intereses, satisfagan las expectativas vitales de sus integrantes y, para el caso de las que son empresas, de sus colaboradores, porque su pleno compromiso solo se alcanzará cuando se presente un entrelazamiento significativo entre sus expectativas vitales y los intereses de aquellas (en términos de Leonardo Schvarstein ${ }^{2}$, que no predomine, para ellas y con respecto al individuo, la imagen y el valor del "personaje" -el rol que se desempeña- ante los de la persona que se es). A este respecto, vale la pena mencionar que algunas teorías administrativas actuales han destacado que solo el pleno compromiso de los colaboradores con la empresa logrará la producción de la plusvalía relativa requerida, pues la obediencia no implica el uso de la libertad, mientras que la responsabilidad la presupone.

De acuerdo con Maturana, la administración, desde lo que él llama la "emoción de la exigencia", demanda por respuesta la obediencia, mientras que la administración, desde la "emoción de la aceptación" del otro, abre el espacio para que cada quien responda por sus actos desde el respeto por sí mismo; es decir, desde el respeto por

2 Concepto expresado durante una de sus intervenciones en el $3^{\text {er }}$ Seminario Internacional Otro Sentido, 2011: "Las conversaciones en las organizaciones", llevado a cabo por la Corporación Colegiatura Colombiana. ( $\mathrm{N}$ de los A). 
los compromisos adquiridos. Por eso, según él, quien obedece no responde por sus actos, mientras que quien actúa en libertad sí lo hace.

Por otro lado, el concepto de "sistema social organizado" no proporciona la explicación de cómo las organizaciones se realizan fácticamente, por qué se extienden como modo de definición de la situación para el comportamiento, cómo se mantienen o por qué desaparecen (Luhmann, 1997: 53).

En términos pragmáticos, con respecto a la comunicación intra y extra-organizacional, los mensajes de las organizaciones son diversos, múltiples y dinámicos, por la naturaleza de las mismas: la comunicación de los mensajes se construye con aquello que la entidad comunica oficialmente y con los diferentes mensajes que se intercambian entre los que la conforman. Ese intercambio de mensajes entre los individuos de la organización, cuya comunicación no es posible uniformar ni controlar, influirá y condicionará el modo de construir los mensajes (Múnera Uribe ES Sánchez Zuluaga, 2003: 351).

En palabras de Manuel Martín Serrano: "El proceso de comunicación solo es posible cuando concurren todos los sistemas que en ella participan. Y siempre que operen todos los componentes, cada uno en el momento que le corresponde" (Serrano, 2007: 153). Así, la comunicación puesta en el hecho del vivir es el resultado del actuar y del pensar conjuntos.

\section{Las implicaciones de la comunicación}

La comunicación es un fenómeno que es transversal a todos los actos humanos; es una categoría de la práctica social; un mecanismo que contribuye a la dinámica evolutiva de esa misma categoría cultural; es, según Martín Serrano, un recurso que desarrollan los homínidos para adaptarse a su desadaptada condición en el mundo natural: "Está implicada en la reproducción de los grupos, los cuales satisfacen las necesidades colectivas, en tanto que la informa- ción que producen sobre el medio social que han creado se oriente a impedir el regreso al estado de naturaleza. Pero al tiempo se utiliza la comunicación para reproducir el estado de naturaleza" (Serrano, 2007: 163).

Desde la ontología lingüística, se ha afirmado que somos seres en el lenguaje y no fuera de él. Sin el lenguaje no tendríamos conciencia de nuestra identidad como seres humanos y, algo mucho peor, solo viviríamos unidos a una realidad temporal y coyuntural; sin él no podríamos desarrollar las emociones. En términos de Humberto Maturana, la realidad existe porque la "empalabramos". Los seres humanos habitamos en el lenguaje. Esta concepción del lenguaje como instrumento generativo es clave para entender la manera de comprender las organizaciones, ya que estas, como cualquier otro fenómeno social, existen porque ocupan un lugar en el lenguaje; en esta medida existe lo que se nombra, y, según Rubén Dittus (s. f.), esta característica es precisamente la que hace posible modificar la realidad social. Desde esta perspectiva, "la investigación de la comunicación tiene el potencial y el deber de enfocarse en agendas de investigación que puedan ayudar a las sociedades y a la gente a 'comunicarse mejor'" Donsbach, (s. f. citado en Fuentes Navarro, 2010: 43).

La comunicación es la interacción mediante la cual los seres vivos acoplan sus conductas al entorno, al mismo tiempo que el entorno adquiere consistencia social. La interacción no solo es una mediación, sino un proceso simbólico que afecta la comprensión del mundo y la forma como se actúa en él a través de los vínculos intersubjetivos establecidos con los otros. Desde el enfoque de Marta Rizo,

.. la interacción comunicativa es un proceso de organización discursiva entre sujetos que, mediante el lenguaje, actúan en un proceso de afectación recíproca. (...) Al margen de qué o quién inicie el proceso de interacción, el resultado es siempre la modificación recíproca de los estados iniciales de los participantes. La interacción es la trama 
discursiva que permite la socialización del sujeto por medio de sus actos dinámicos, su adaptación al entorno y la comprensión de las acciones propias y ajenas" (Rizo, s. f.).

Los grupos humanos, las comunidades, entendidas como organizaciones, mediaciones e interacciones, son, en sí, redes de conversaciones que se nutren de una determinada cultura. "Las organizaciones son grupos humanos complejos. Son delimitaciones de la sociedad misma, en las cuales coexisten diferentes individuos con diferentes búsquedas pero con la posibilidad de confluir en algunos objetivos comunes. En la organización se presentan interacciones y transacciones que llevan a la cristalización o al fracaso del proyecto institucional y, por ende, de su acción en la sociedad" (Múnera Uribe E Sánchez Zuluaga, 2003: 187). Las organizaciones son pues, un sistema estructurado y en evolución, cuyos esfuerzos coordinados tienen por objeto alcanzar metas en ambientes dinámicos, generan identidad; comparten, según Rafael Echeverría, en su libro Ontología del lenguaje, un pasado, una forma colectiva de hacer las cosas en el presente y un sentido común de dirección hacia el futuro.

El reconocimiento del papel generativo del lenguaje permite un nuevo enfoque para la comprensión de las organizaciones en general, como asimismo de las empresas y las actividades gerenciales y de management (...) Una empresa es una red estable de conversaciones que genera una identidad en el mundo que trasciende a sus miembros individuales. (...) Las empresas son agentes de acción y, en cuanto tales, se vuelven socialmente responsables de sus actos (Revah, s. f.).

Desde la mirada de Luz Gabriela Gómez,

.. la comunicación organizacional integra, da vida y acción a la empresa; es la metodología de la administración. Es la fuerza que amarra a las personas con los propósitos compartidos. Sin comunicación no existe interacción coordinada; cuando falla la comunicación también falla la actividad organizada. Desde la comunicación se mezclan los pensamientos analítico y creativo, para hacer posibles los proyectos (Gómez, 2003: 38).

La organización y la comunicación se encuentran estrechamente vinculadas dentro del funcionamiento que busca resultados positivos entre necesidades y satisfactores. Sus vínculos, según María Asunción Anca, vienen dados a través de los componentes (Anca García, s. f.): a) Políticos, de una acción estratégica global; b) Socioculturales, de los valores y de las representaciones simbólicas de las acciones reguladas por normas; y c) Psicológicos, los establecidos mediante las acciones dialógicas entre los participantes o miembros de la organización. En esta misma línea, la psicóloga Susana de la Iglesia argumenta que como la palabra "conversar" viene del latín "conversus", que significa "convertirse", a medida que vamos conversando nos convertimos en alguien distinto, nos transformamos a través de la palabra. Una persona nunca será la misma después de haber conversado con otra; un equipo de trabajo se transforma de acuerdo con las conversaciones entre sus miembros; esto es porque las conversaciones no solamente modifican las acciones, sino también porque los resultados modifican a la persona misma. Una organización es lo que es porque las personas que la forman son quienes son.

En palabras de Pablo Múnera: "El primer y gran reto que se le impone al comunicador empresarial es reivindicar la bella etimología de la comunicación (viene del latín comunicare: hacer común). (...) El hombre no es, pues, totalmente egoísta, ni únicamente social. Es en relación con los otros. La identidad se construye con la alteridad. (...)" (Múnera, 2005: 72-73). Y continúa: "A Georges Gusdorf se le debe una de las concepciones más profundas y sintéticas de la comunicación: el proceso de la búsqueda del tú por parte del yo para confluir en el nosotros. Una tensión entre el mundo interior y el mundo exterior de la persona; o como lo plantea Freud, entre individuo y sociedad, entre natura y cultura" (Múnera, 2005: 73). 
La comunicación, las organizaciones y las conversaciones son cuestiones que involucran el yo, el otro y el nosotros como conceptos antropológicos, psicológicos y sociológicos. "El yo, al iniciar un proceso comunicativo, tiene la intensión y le apuesta al nosotros. Ahí comienza el juego de seducción y negociación para hacer que el tú se involucre en el proceso, para que se comparta una sociedad bajo el apelativo plural: nosotros. Aquí comienza la comunidad (hacer común)" (Múnera, 2005: 73).

\section{Un estado del arte de las conversaciones organizacionales como objeto fenomenológico}

Como lo menciona Dittus (s. f.): "la perspectiva presentada [la organización, definida como un sistema autopoiético de conversaciones e imaginarios] describe el establecimiento de un nuevo paradigma en el ámbito organizacional". Y continúa: "Se ha entendido en los últimos años que es imposible comprender el funcionamiento de una organización si no se toma en cuenta su realidad comunicativa, ya que son las prácticas lingüísticas de las personas las que definen su identidad" (Dittus, s. f.). Queda así establecida la importancia del enfoque teórico que considera a las organizaciones como determinadas por las conversaciones que se llevan a cabo en su interior.

Por otra parte, ya que el mismo Peter Drucker, padre de la Administración, advertía desde tiempo atrás sobre la relevancia de las conversaciones como componentes de la dinámica empresarial, autores con este enfoque, como Peter Senge, Walden Bello, Ray Stata, o Rafael Echeverría, han indagado y opinado sobre el tema en tiempos más recientes (el concepto de las "conversaciones inteligentes" es fruto de ello, por ejemplo). Sin embargo, con referencia a la fenomenología ${ }^{3}$ propia y específica de las con-

3 La Fenomenología, al igual que la Etnografía, es una metodología para indagar las verdades del hombre en su cotidianidad. El término fenomenología fue usado por primera vez por Lambert para significar, en un sentido amplio, la "ciencia de los fenómenos". Puesto que los objetos se nos revelan en la conciencia, se llama Fenomenología, en acepción estricta, a la ciencia de los versaciones como actos comunicativos dentro de las empresas y otro tipo de organizaciones, es la teoría psicológica canadiense del Análisis Transaccional la que más ha influido en el ámbito académico al tratar de dar cuenta de ella. En el contexto colombiano, se destaca en esa línea el aporte que constituye la obra de Gilbert Brenson, renombrado psicólogo estadounidense, discípulo de Eric Berne, fundador del Análisis Transaccional, quien ha vivido y trabajado en Bogotá durante años (es notoria su aplicación de los conceptos de "empoderamiento" del lenguaje y de la "red holárquica", en el ambiente comunicativo organizacional).

Para el caso concreto de Medellín, se evidencia la obra escrita por Pablo Antonio Múnera y Uriel Sánchez Zuluaga, Comunicación empresarial: Una mirada corporativa, para la Asociación Iberoamericana de Comunicación Estratégica (AICE), de 2003, en donde, aunque no es el tema principal, se abordan las conversaciones como partícipes en la construcción y transmisión de mensajes organizacionales. En cuanto a las investigaciones, se han realizado algunas (la fuente de referencia es la Facultad de Comunicación Social-Periodismo de la Universidad Pontificia Bolivariana), exclusivamente en la modalidad de trabajos de grado, donde se evidencia la ausencia de estudios con respecto al tema de las conversaciones, en donde este sea el objeto específico. El interés se ha centrado en otros aspectos de la comunicación organizacional, tales como los blogs como forma de comunicación tecnológica -análoga (como una especie de metáfora) al "conversar"-, pero sin abordar el fenómeno de las conversaciones en sentido estricto ("A bloguear, es hora de conversar con los públicos. Los blogs en la comunicación organizacional", de Martha Lucía Gómez, trabajo de grado, 2009); las "reuniones" como parte de los actos propios de la comunicación organizacional, en forma genérica, sin hacer alusión alguna al concepto de "con-

fenómenos que se manifiestan en dicha instancia. Fue fundada formalmente por Edmund Husserl, a fin de lograr una base inatacable para todas las ciencias. (N de los A). 
versación", ni incluirlo (Rituales empresariales: elementos de comunicación, identidad y expresión cultural, de Ana Isabel Martínez, trabajo de grado 2000); y la comunicación interpersonal y la telefónica como modalidades de comunicación intraempresarial (sin ahondar en el asunto, ni mencionar las conversaciones como objeto de estudio) en la empresa ICONTEC, como estudio de caso elegido ("Diseño y creación de estrategias internas de comunicación, propias para una empresa de servicios: ICONTEC Regional Medellín", de Juliana Vélez y Alejandra Rúa, trabajo de grado, 2003). Los trabajos mencionados definen los canales, las estrategias, las interacciones, los procesos comunicacionales, y la transmisión de mensajes como factores para el mejoramiento de las relaciones organizacionales.

Los antecedentes descritos hacen relevante la realización de investigaciones en las que el objeto específico sea la conversación organizacional, puesto que en el ámbito mundial, aunque el interés se ha centrado en los fenómenos de interrelación y comunicación organizacional en general (sobre los cuales se encuentra abundante literatura que, en la mayoría de los casos, es producto de investigaciones al respecto), el conocimiento recopilado sobre las conversaciones organizacionales, específicamente, y cómo éstas se llevan a cabo -y en concreto las de índole empresariales escaso y constituye un campo nuevo de indagación en el ámbito nacional e internacional, como ha podido constatarse.

Por último, y ante lo descrito anteriormente, podrían considerarse algunos interrogantes que formula Alejando Formanchuk (s. f.), como posibles puntos de partida para la indagación que se propone:

¿Qué pasa con la comunicación interna en un ambiente marcado por el hacer, el deshacer y el rehacer constantes, donde la sociabilidad baila al ritmo del desarraigo y la movilidad?

¿Podemos aún pensar la comunicación interna desde una dimensión humana o estamos limitados a trabajar con fragmentos de sujetos móviles, diversos, esporádicos?

¿Cómo recuperar una perspectiva global de la comunicación en la empresa cuando lo que está en juego es una nueva sensibilidad?

\section{Referencias bibliográficas}

Alonso-Cortés Fradejas, M.D. (1998). Gramática y conversación: constantes gramaticales del género y fenómenos estructurales. León: Universidad de León.

Anca García, M.A. (s.f.). Fundamentos teóricos del constructivismo pedagógico (On line). Consultado el 7 de junio de 2011. <http://www.monografias.com/ trabajos27/constructivismopedagogico/constructivismo-pedagogico.shtml>

Burke, P. (2002). De Gutemberg a internet: una historia social de los medios de comunicación. Madrid: Taurus.

Dittus, R. (s.f.). El giro lingüístico y sus efectos en la comunicación organizacional. Documento PDF (On line). Acceso: junio 1 de 2011. <http://www. periodismoudec.cl/estudiosdeperiodismo/index 2 . php?option $=$ com _ contentEdo ${ }_{-}$pdf $=1 \mathcal{E} \mathrm{id}=59>$

Facultad de Comunicación y Lenguaje de la Pontificia Universidad Javeriana - Bogotá y Departamento de Comunicación y Lenguaje de la Pontificia Universidad Javeriana - Cali (2010). Revista Signo y Pensamiento, 29 (57)

Formanchuk, A. (s.f.). Comunicación interna y sentido de pertenencia: cuando la velocidad corta raíces. Revista Electrónica Ser humano y Trabajo. Promoviendo el desarrollo del talento humano (On line), Buenos Aires, Argentina. Acceso: octubre 19 de 2011. $<$ http://www.sht.com.ar/archivo/temas/pertenencia.htm>

Gómez, L.G. (2003). Comunicación, indagación y ciudad. Medellín: UPB.

Luhmann, N. (1997). Organización y decisión, autopoiesis, acción y entendimiento comunicativo. Barcelona: Anthropos. 
Maturana Romesín, H. (2002). Transformación en la convivencia, $2^{\mathrm{a}}$ ed. Documento PDF (On line) Acceso: noviembre 8 de 2011. <http:/books.google. com. $\mathrm{co} /$ la + historia+evolutiva + de + nuestro+linaje $+\mathrm{co}$ mo+una+historia+de+la+conversación $>$

Múnera Uribe, P.A. \& Sánchez Zuluaga, U.H. (2003). Comunicación empresarial: una mirada corporativa. Medellín: Asociación Iberoamericana de Comunicación Estratégica AICE -

Múnera, P.A. (2005). El comunicador corporativo: entre la teoría y la práctica. De la etimología de la comunicación a la organización viable. Medellín: Zuluaga.

Platón. (2003). Diálogos. Obras completas. Madrid: Gredos.

REVAH, José Luis. Diseño de conversaciones en las organizaciones. Una nueva experiencia educativa. (On line).
Consultado el 7 de junio de 2011. <http://blog. revah.com.ar/?p=10>

Rizo, M. (s.f.). El interaccionismo simbólico y la escuela de Palo Alto. Hacia un nuevo concepto de comunicación. Portal de la Comunicación. Documento PDF (On line). Acceso: junio 8 de 2011. <http://www.portalcomunicacion. com/esp/pdf/aab>

Serrano, M.M. (2007). Teoría de la comunicación. La comunicación, la vida y la sociedad. Madrid: McGraw-Hill.

Van Dijk, T.A. (s.f.). La ciencia del texto, $3^{a}$ ed. Documento PDF (On line). Acceso octubre 10 de 2011. <http:// www.discursos.org/oldbooks/Teun\%20A\%20 van\%20Dijk\%20\%20La\%20Ciencia\%20del\%20> 